\title{
Producción, calidad de leche y beneficio:costo de suplementar vacas holstein con Tithonia diversifolia ${ }^{1}$
}

\section{Milk production, quality and benefit:cost ratio of supplementing Holstein cows with Tithonia diversifolia}

\author{
Luis Alberto Gallego-Castro², Liliana Mahecha-Ledesma², Joaquín Angulo-Arizala²
}

\begin{abstract}
Resumen
El objetivo de este trabajo fue analizar la producción, la calidad de leche y la relación beneficio:costo de la inclusión de tres niveles $(0 \%, 15 \%$ y 25\%) de Tithonia diversifolia en el suplemento de vacas holstein. En un sistema de producción bovina de leche del trópico alto se utilizaron nueve vacas (70 a 120 días de lactancia y dos a cinco partos), distribuidas aleatoriamente en tres grupos que pastoreaban praderas de kikuyo (Cenchrus clandestinus), en la hacienda La Montaña, San Pedro de los Milagros, Antioquia, Colombia, a $2350 \mathrm{msnm}$, durante marzo de 2015. A los 56 días se cosechó T. diversifolia (girasoles mexicanos) de un banco forrajero ubicado a $2456 \mathrm{msnm}$, se secó en marquesinas durante diez días aproximadamente, fue molido y almacenado para la formulación de suplementos isoenergéticos (1,8 Mcal ENL/kg) e isoproteicos (14\% PC). Se determinó el consumo de materia seca (CMS), la producción de leche corregida 4\% grasa, composición de la leche (proteína, grasa, lactosa, sólidos totales, nitrógeno ureico en leche -MUN-), conteo de células somáticas (RCS), la relación leche producida:suplemento y la relación beneficio:costo. Hubo diferencias significativas con la inclusión de $25 \%$ de harina de $T$. diversifolia para lactosa, MUN, RCS, relación leche producida:suplemento consumido y relación beneficio:costo; no así para CMS, leche corregida al $4 \%$ de grasa, proteína, grasa y sólidos totales. La inclusión de $25 \%$ de botón de oro en el suplemento alimenticio para vacas lecheras en pastoreo mejoró la calidad de leche referente a concentración de lactosa, eficiencia en la utilización del suplemento y relación beneficio:costo.
\end{abstract}

Palabras clave: forrajeras arbustivas, harina de botón de oro, vacas lecheras, alimentación animal.

\begin{abstract}
The aim of this study was to analyze the milk production, quality and cost:benefit ratio the addition of three levels $(0 \%, 15 \%$ and $25 \%)$ flour of Tithonia diversifolia in the supplement of Holstein cows. In a bovine milk production system in a high tropic were used nine cows ( 70 to 120 milk days and parity two to five). They were randomly assigned in three groups, which grazed on Kikuyo grass (Cenchrus clandestinus) in La Montaña farm, located in San Pedro de los Milagros, Antioquia, Colombia, at 2350 meters above sea level. This study was carried out during March 2015. At 56 days, T. diversifolia (wild sunflower) from a fodder bank located at 2456 masl was harvested, and dried using marquees ground for an approximate period of time of ten days, it was then minced and stored to prepare isoenergetics $(1.8 \mathrm{Mcal} \mathrm{NEL} / \mathrm{kg})$ and isoproteics $(14 \% \mathrm{CP})$ supplements. The study evaluated its effect on dry matter intake (DMI), corrected milk production ( $4 \%$ fat, FCM), milk composition (protein, fat, lactose, total solids, milk
\end{abstract}

\footnotetext{
Recibido: 27 de setiembre, 2016. Aceptado: 16 de enero, 2017. Este trabajo formó parte de la tesis de grado de la maestría en Ciencias Animales del primer autor, realizada en la Facultad de Ciencias Agrarias de la Universidad de Antioquia, Colombia.

2 Universidad de Antioquia, Facultad de Ciencias Agrarias, Grupo de investigación en Ciencias Agrarias - GRICA,. AA 1126, Medellín, Colombia.luis.gallego@udea.edu.co, liliana.mahecha@udea.edu.co, joaquin.angulo@udea.edu.co (autor para correspondencia).
} 
urea nitrogen -MUN), somatic cell count (SCC), produced milk: supplement intake and cost:benefit ratio. The results showed significant differences for the inclusion of $25 \%$ of $T$. diversifolia flour in the supplement for benefit:cost ratio, lactose, MUN, SCC and produced milk:supplement intake ratio, contrary to what happened with DMI, corrected $4 \%$ fat milk production, protein, fat and total solids that not differ. The addition of $25 \%$ of wild sunflower in the supplement for grazing dairy cows improved the milk quality in reference to lactose concentration, efficiency in the use of the supplement, cost:benefit ratio.

Keywords: forage shrubs, wild sunflower flour, dairy cows, animal feeding.

\section{Introducción}

Los sistemas especializados de producción de leche con razas Bos taurus en Colombia, se desarrollan básicamente bajo condiciones de trópico de altura $(1800$ a $3000 \mathrm{msnm})$ y precipitaciones entre 1600 a $2200 \mathrm{~mm}$ (Morales et al., 2013), donde las pasturas generalmente presentan altos contenidos de humedad (86\% a $88 \%$ ) y baja calidad nutricional (Boschini y Pineda, 2016). Los productores suministran suplementos que ayudan a mejorar la dieta de los animales; la cual consta principalmente de una base forrajera de monocultivos de kikuyo (Cenchrus clandestinus), con déficit de nutrientes, por lo que no satisfacen los requerimientos de los animales (Correa et al., 2012; Morales et al., 2013).

Es necesario que la oferta de suplementos para estos sistemas ganaderos se diversifique, con la intención de mejorar el balance de nutrientes ofertados, y a la vez, la calidad del producto obtenido, disminuyendo los costos de producción y los impactos sobre el ambiente (Bayati et al., 2013).

El manejo de la alimentación requiere de una utilización eficiente de los recursos disponibles (Mahecha et al., 2002), que permita mejorar el balance nutricional de los animales. En la actualidad, la composición de los suplementos alimenticios está basada en materias primas como el maíz, subproductos de la soya y de la transformación e industrialización de cereales, materiales que en su generalidad no logran que se alcancen todos los resultados esperados en lo productivo, lo económico y en la salud de los animales.

La inclusión de materiales alternativos que promuevan una mejor estabilidad de la actividad fermentativa ruminal, puede lograr una mayor eficiencia en la utilización de los alimentos e influir en los resultados productivos de las lecherías especializadas (Correa et al., 2012).

Actualmente se han estudiado diferentes propuestas para diversificar la alimentación de los vacunos en estos sistemas de producción, las cuales promueven la implementación de bancos forrajeros, donde se destaca el botón de oro (Mahecha et al., 2007). Esta especie forrajera puede constituir una alternativa en la alimentación de ganado bovino de producción lechera en condiciones de trópico alto, bien sea mediante sistemas de corte y acarreo o de procesamiento, que permita la obtención de harinas que se empleen en la preparación de los suplementos alimenticios.

La Tithonia diversifolia (Hemsl A. Gray), planta de la familia Asteraceae, conocida como botón de oro, falso girasol, mirasol, margaritón, entre otros, y originaria de América Central, ha sido introducida en el trópico en casi todo el mundo (Maina et al., 2012). Esta especie tiene cualidades tales como: alta producción de biomasa, que llega a las 19 t/ha/MS al año (Gallego et al., 2015), plasticidad ecológica, recuperación de suelos degradados, resistencia a plagas y enfermedades, y usos en alimentación de diferentes especies animales, lo que permiten clasificarla como planta forrajera de un alto potencial para la producción animal. Asimismo, se ha indicado su fácil adaptación a diversas condiciones de suelos y resistencia al corte frecuente (Nieves et al., 2011).

La calidad nutricional de botón de oro puede variar en función de la edad de cosecha, se ha señalado como buen momento alrededor de las ocho semanas. Las prácticas culturales realizadas, las condiciones del suelo donde se cultive y el efecto de las temporadas secas o lluviosas a lo largo del año, tendrán influencia sobre su calidad 
nutricional (Lugo et al., 2012), alcanzando niveles de proteína de un 25,4\% y 25,2\% de fibre detergente neutro (FDN) (Rivera et al., 2015), valores que, sin embargo, pueden ser muy variables de acuerdo con lo antes mencionado.

T. diversifolia ha sido reconocida por su contenido proteico y, aunque este puede presentar importantes variaciones, una importante fracción es proteína verdadera; este forraje contiene 48,37\% de aminoácidos esenciales en relación con la proteína total (Macías y Martínez, 1997); además, presenta un buen contenido de carbohidratos solubles (Medina et al., 2009), niveles de fósforo $(0,36 \%)$ y calcio $(3,17 \%)$ superiores a los encontrados en muchos otros forrajes (Medina et al., 2009), y su nivel de taninos no es tan alto como para llegar a influenciar de manera negativa en el aprovechamiento de los nutrientes de la planta por parte del ganado bovino (Gualberto et al., 2010).

El botón de oro ha sido utilizado por productores, principalmente en sistemas de pastoreo directo o para corte y acarreo. Cuando se evaluó el efecto de la inclusión del forraje fresco de T. diversifolia como reemplazo parcial del alimento concentrado en vacas cruzadas holstein por cebú, no se encontraron diferencias en la producción (12,41 $\mathrm{kg} / \mathrm{día}$ ) y la calidad de la leche (3,67\% de grasa y 3,32\% de proteína) (Mahecha et al., 2007). T. diversifolia puede llegar a convertirse en una materia prima en la elaboración de alimentos balanceados. Los suplementos comerciales son muy utilizados en las ganaderías de alta producción lechera, con altos costos, dado que cereales como el maíz y los subproductos de oleaginosas como la torta de soya, presentan precios de mercado cada vez más elevados; por lo que, el uso de materiales como el botón de oro puede llegar a realizar aportes importantes en la mejora de la competitividad y sostenibilidad de estos sistemas en las zonas de trópico alto colombiano.

El objetivo de este trabajo fue analizar la producción, calidad de leche y la relación beneficio:costo de la inclusión de tres niveles $(0 \%, 15 \%$ y $25 \%)$ de $T$. diversifolia Hemsl. A Gray en el suplemento de vacas holstein.

\section{Materiales y métodos}

El proyecto contó con el aval del Comité de Ética para la Experimentación Animal de la Universidad de Antioquia, mediante acta No 83 de mayo de 2013.

La fase de experimentación se desarrolló durante marzo de 2015, en ella se analizó el efecto de la inclusión de botón de oro en el suplemento alimenticio sobre el comportamiento productivo de vacas lecheras, proceso que se llevó a cabo en la hacienda La Montaña, propiedad de la Facultad de Ciencias Agrarias de la Universidad de Antioquia, Colombia. La hacienda se encuentra ubicada en el municipio de San Pedro de los Milagros (norte del departamento de Antioquia), a $2350 \mathrm{msnm}$, con temperatura promedio de $14{ }^{\circ} \mathrm{C}$, humedad relativa del $72 \%$ y precipitación promedio anual de $1575 \mathrm{~mm}$, lo que la clasifica en la zona de vida de bosque Húmedo Montano Bajo (bh-MB).

Para la obtención de la harina de botón de oro se mantuvo el cultivo bajo condiciones de trópico alto, en la finca Santa Martha, localizada en el municipio de Guarne (Antioquia, Colombia) a $2453 \mathrm{msnm}$, la plantación no recibió ningún tratamiento de fertilización durante el periodo de crecimiento, se desmalezó manualmente, y cuando llegó a los $30 \mathrm{~cm}$ de altura, se realizó un corte de uniformización. Después de 56 días de rebrote, en el mes de enero de 2015, se cosecharon plantas completas (hojas y tallos) que fueron secadas en marquesinas durante diez días aproximadamente; este material se molió y empacó para su posterior uso.

La harina de botón de oro se incluyó en niveles de 15\% y 25\% y sustituyó parcialmente otros ingredientes de uso tradicional en la finca, de manera que se obtuvieron formulaciones isoproteicas e isoenergéticas (Cuadro 1), de acuerdo con las características del suplemento utilizado en la hacienda cotidianamente para el lote de vacas de alta producció (1,8 $\mathrm{Mcal} / \mathrm{kg} \mathrm{EN}_{\mathrm{L}}$ y $\left.14 \% \mathrm{PC}\right)$.

A los alimentos concentrados se les realizó análisis químico proximal de acuerdo con AOAC (2005), mientras que le $\mathrm{EN}_{\mathrm{L}}$ se estimó según las ecuaciones de predicción planteadas por Buxadé (1995), a partir de la energía bruta (EB), determinada por calorimetría. Las muestras fueron analizadas en el Laboratorio Integrado de Nutrición Animal, Bioquímica y de Pastos y Forrajes de la Facultad de Ciencias Agrarias de la Universidad de Antioquia. La 
Cuadro 1. Ingredientes utilizados en la preparación de los alimentos concentrados de uso tradicional en la lechería de la hacienda La Montaña, con inclusión de $0 \%(\mathrm{C} 0), 15 \%(\mathrm{C} 15)$ y $25 \%(\mathrm{C} 25)$ de harina de Tithonia diversifolia. Antioquia, Colombia. 2015.

Table 1. Ingredients used in the formulation of feed concentrate used traditionally in the dairy farm "La Montaña", including 0\% (C0), 15\% (C15) and 25\% (C25) of Tithonia diversifolia flour. Antioquia, Colombia. 2015.

\begin{tabular}{lccc}
\hline Materia prima & C0* & C15 & C25 \\
\hline Botón de oro & 0,00 & 15,00 & 25,00 \\
Maíz amarillo & 38,82 & 40,00 & 40,00 \\
DDGS Golden (maíz) & 18,00 & 5,00 & 10,18 \\
Maíz extruido & 15,00 & 14,41 & 0,00 \\
Salvado de trigo & 12,95 & 5,00 & 10,77 \\
Melaza & 5,00 & 5,00 & 5,00 \\
Semilla de algodón & 4,00 & 4,00 & 4,00 \\
Harina de pescado & 2,00 & 2,00 & 2,00 \\
Torta de soya 47 & 1,37 & 6,57 & 0,00 \\
Carbonato de calcio & 1,36 & 1,52 & 1,54 \\
Sal de mar & 0,80 & 0,80 & 0,80 \\
Premezcla & 0,50 & 0,50 & 0,50 \\
Adsorbente micotoxinas ${ }^{1}$ & 0,20 & 0,20 & 0,20 \\
Total & 100,00 & 100,00 & 100,00 \\
\hline
\end{tabular}

* Composición tradicional de la dieta empleada en la finca con $1,8 \mathrm{Mcal} / \mathrm{kg}_{\mathrm{EN}} \mathrm{y}$ $14 \%$ PC; las que incluyen botón de oro se balancearon bajo los mismos parámetros / Traditional diet composition used in the farm with 1,8 Mcal $/ \mathrm{kg} \mathrm{EN}_{\mathrm{L}}$ and $14 \% \mathrm{PC}$; diets including mexican sunflower were balanced under similar parameters.

${ }^{1}$ Aluminosilicato de calcio y sodio hidratado / Hydrated sodium calcium aluminosilicate.

composición presentada puede considerarse isoenergética e isoproteica, dado que cuando la diferencia de energía o de proteína entre dietas es inferior al 10\%, los contenidos de estos compuestos se consideran similares (S. Posada, comunicación personal, 2016). La composición nutricional de las tres dietas evaluadas, del pasto kikuyo y de la harina de botón de oro, definida mediante análisis bromatológico y ENL predicha, se presenta en el Cuadro 2.

El suplemento se suministró en dos ocasiones por día (5:00 a.m. y 2:00 p.m), en cada uno de los ordeños, a razón de un kilogramo por cada tres litros de leche producida, a partir del octavo litro, según las normas establecidas en la finca. Las vacas fueron manejadas en un sistema de pastoreo rotacional en franjas de pasto kikuyo (Cenchrus clandestinus).

\section{Consumo de materia seca}

Para determinar el consumo de forraje en pastoreo se utilizó como indicador externo el óxido de cromo $\left(\mathrm{Cr}_{2} \mathrm{O}_{3}\right)$, del que se suministraron $20 \mathrm{~g} / \mathrm{vaca}$ /día, y como marcador interno se utilizó la lignina detergente ácido (Lachmann et al., 2003). El óxido de cromo se suministró durante doce días, los primero siete días fueron de acostumbramiento, para los posteriores cinco realizar la colecta de heces de manera individual; al finalizar se tomó una muestra de las 
Cuadro 2. Composición bromatológica de la harina de botón de oro, el pasto kikuyo y las dietas de uso tradicional en la lechería de la hacienda La Montaña, con diferentes niveles de harina de botón de oro $(0 \%, 15 \%$ y $25 \%)$ y del pasto kikuyo, empleadas en la alimentación de las vacas. Municipio de San Pedro de los Milagros, Antioquia, Colombia. 2015.

Table 2. Bromatological composition of the mexican sunflower flour, kikuyo grass and traditional diets used in the dairy farm "La Montaña", including differents levels of mexican sunflower (0\%, 15\% and 25\%) and Kikuyo grass, used for feeding the cows. Municipality of San Pedro de los Milagros, Antioquia, Colombia. 2015.

\begin{tabular}{lccccc}
\hline Nutriente & Co & C15 & C25 & $\begin{array}{c}\text { Harina de botón } \\
\text { de oro }\end{array}$ & Kikuyo \\
\hline Proteína cruda & 13,31 & 12,36 & 13,19 & 11,66 & 18,45 \\
Energía bruta (EB) & 3797 & 3765 & 3748 & 3618 & 4219 \\
Energía neta de lactancia (EN $)^{*}$ & $1,82^{*}$ & $1,80 *$ & $1,79 *$ & $1,43 * *$ & $1,25 * * *$ \\
Fibra detergente neutro (FDN) & 15,87 & 17,91 & 25,68 & 37,17 & 61,51 \\
Fibra detergente ácido (FDA) & 7,48 & 8,35 & 14,37 & 32,40 & 28,91 \\
Lignina & 0,42 & 0,84 & 2,51 & 6,02 & 1,68 \\
Cenizas & 8,40 & 7,80 & 9,45 & 12,14 & 10,06 \\
Calcio & 2,21 & 1,26 & 1,92 & 2,34 & 1,20 \\
Fósforo & 0,30 & 0,31 & 0,33 & 0,22 & 0,31 \\
Extracto etéreo & 3,61 & 3,43 & 3,18 & 2,96 & 7,90 \\
Humedad & 11,83 & 11,76 & 13,04 & 14,22 & 13,38 \\
\hline
\end{tabular}

* Buxadé (1995): 3,8 Mcal EB =3,22 Kcal $\mathrm{ED}$ y $\mathrm{EN}_{\mathrm{L}}=0,677 * \mathrm{ED}-0,359$.

** Buxadé (1995): 2,23 - 0,0216* FDN.

*** Buxadé (1995): 2,86-0,0262* FDN.

heces de cada vaca y se llevaron al Laboratorio de Análisis Químico y Bromatológico de la Universidad Nacional de Colombia, sede Medellín, para determinar la concentración de cromo. A partir de lo cual se estimó la producción total de heces $(\mathrm{PH})$, de acuerdo con el siguiente modelo:

$\mathrm{PH}=\frac{\mathrm{g} \mathrm{Cr} / \mathrm{vaca} / \text { día }}{\mathrm{g} \mathrm{Cr} / \mathrm{kg} \mathrm{de} \mathrm{heces}}$

Las muestras de heces colectadas del forraje y de los suplementos suministrados a las vacas, fueron llevadas a Laboratorio Integrado de Nutrición Animal, Bioquímica y de Pastos y Forrajes de la Facultad de Ciencias Agrarias de la Universidad de Antioquia, para determinar contenido de lignina detergente ácido (LDA).

Se determinó el consumo de materia seca (CMS) con el siguiente modelo, dado que los animales recibieron suplemento, $\mathrm{CMS}=\left(\left(\mathrm{PH} * \mathrm{LDA}_{\mathrm{H}}\right)-\left(\mathrm{CS} * \mathrm{LDA}_{\mathrm{c}}\right)\right) / \mathrm{LDA}_{\mathrm{f}}$.

Donde:

$\mathrm{PH}=$ producción heces $(\mathrm{kg} \mathrm{MS})$.

$\mathrm{LDA}_{\mathrm{H}}=$ lignina detergente ácido de las heces.

$\mathrm{CS}=$ consumo suplemento.

$\mathrm{LDA}_{\mathrm{c}}=$ lignina detergente ácido del suplemento balanceado.

$\mathrm{LDA}_{\mathrm{f}}=$ lignina detergente ácido del forraje. 
Por su parte, el consumo de suplemento individual se registró diariamente; el consumo individual de la fase pre-experimental se tomó como covariable.

\section{Cantidad y calidad de leche}

La producción total de leche se determinó por la cantidad diaria individual (dos ordeños), se tomó la producción antes de iniciar la fase experimental como covariable. Se tomaron muestras que fueron enviadas al Laboratorio de Calidad de Leches de la Facultad de Ciencias Agrarias de la Universidad de Antioquia, donde se analizó la calidad química (grasa, proteína, lactosa, sólidos totales y nitrógeno ureico en leche (MUN) por espectroscopia infrarroja) y microbiológica (recuento de células somáticas por citometría de flujo). Se realizó el ajuste de producción de leche al $4 \%$ de grasa $(\mathrm{LCG}=[0,4 * \mathrm{~kg}$ leche $]+[15 * \mathrm{~kg}$ grasa $])(\mathrm{NRC}, 2001)$, lo que permitió una mejor comparación de la producción. Para evaluar la calidad de leche se tomaron cuatro mediciones individuales durante la fase experimental, y se estimó la cantidad de grasa y proteína producidas. Se tomó la calidad de leche en la fase pre experimental como covariable.

\section{Relación beneficio:costo}

Para determinar la relación beneficio:costo en cada tratamiento, se consideraron los costos y cantidad del suplemento alimenticio suministrado por grupo de animales según el tratamiento asignado. Para determinar el costo del suplemento se cuantificaron las materias primas convencionales, más la harina de botón de oro (Tithonia diversifolia). Asimismo, se estableció una relación entre la cantidad de leche producida y la cantidad de suplemento consumido, lo que permitió estimar la eficiencia productiva de los animales por tratamiento. Para el cálculo del beneficio obtenido se incluyeron los ingresos por ventas y se empleó el precio básico por kilogramo de leche, establecido por la empresa comercializadora en la región, sin considerar ninguna bonificación.

Para el ensayo se seleccionaron nueve vacas holstein adultas, que tenían entre dos y cinco partos y entre 70 y 120 días de paridas, parámetros que se emplearon para su estratificación, luego fueron distribuidas de manera aleatoria en los tres grupos de estudio. Se determinó consumo de materia seca (CMS), producción de leche (leche corregida $4 \%$ grasa y composición referente a proteína, grasa, lactosa, sólidos totales, MUN, número de células somáticas (RCS), relación leche producida:suplemento y relación beneficio:costo.

Se midieron el CMS, la producción de leche y su composición química en el periodo pre experimental y mediante un análisis de covarianza, se determinó la relación de estas variables antes y después del ensayo.

Se empleó un diseño completamente aleatorizado, se realizó el ANOVA, lo que permitió establecer diferencias entre las medias de las variables evaluadas en cada tratamiento, lo que fue probado mediante prueba Tukey $(\mathrm{P}<$ 0,05), mediante análisis con el programa estadístico SPSS (Statistics 22).

\section{Resultados y discusión}

\section{Consumo de materia seca}

La materia seca consumida estuvo representada por el consumo voluntario de forraje durante el pastoreo y por el suplemento suministrado durante el ordeño de manera individual. El consumo inicial de suplemento se ajustó durante la fase pre-experimental, los resultados se muestran en el Cuadro 3. El consumo previo fue considerado como covariable del consumo obtenido durante la fase experimental; con una relación altamente significativa, con diferencias de consumo de $1,26 \mathrm{~kg}, 0,72 \mathrm{~kg}$ y $0,46 \mathrm{~kg}$ entre las fases pre-experimental y experimental, para $\mathrm{C} 0, \mathrm{C} 15 \mathrm{y}$ 
Cuadro 3. Consumo de suplemento en fases pre-experimental y experimental, y consumo de materia seca total de forraje en fase experimental, por vacas holstein en el municipio de San Pedro de los Milagros, Antioquia, Colombia. 2015.

Table 3. Consumed supplement in pre-experimental and experimental periods, and dry total matter of forage consumed in experimental period, by Holstein cows in the Municipality of San Pedro de los Milagros, Antioquia, Colombia. 2015.

\begin{tabular}{llccc}
\hline & & C0 & C15 & C25 \\
\hline Consumo suplemento (kg MS) & Fase pre-experimetal & $6,56 \mathrm{a}$ & $5,74 \mathrm{a}$ & $5,49 \mathrm{a}$ \\
& Fase experimental & $7,82 * \mathrm{a}$ & $6,46 * \mathrm{a}$ & $5,95 * \mathrm{a}$ \\
\multirow{2}{*}{ Consumo de materia seca } & Materia seca forraje & $11,75 \mathrm{a}$ & $15,32 \mathrm{a}$ & $13,78 \mathrm{a}$ \\
& Materia seca total & $18,64 \mathrm{a}$ & $21,02 \mathrm{a}$ & $18,95 \mathrm{a}$ \\
\hline
\end{tabular}

Letra diferente en la misma fila indican diferencia significativa $(\mathrm{p}<0,05)$ / Different letter in the same row indicate significant differences $(\mathrm{p}<0.05)$.

* Diferencia significativa con su covariable de la fase pre-experimental / Significant difference with covariable of the pre-experimental period.

$\mathrm{C} 25$, respectivamente, se definió que el consumo de suplemento en la fase experimental estuvo influenciado de manera directa por el consumo encontrado en la fase pre-experimental $(P=0,001)$, debido en gran medida a la atención y manejo más preciso de la suplementación en comparación con la rutina diaria de la finca.

El consumo de materia seca (CMS) estimado mediante marcadores, incluyendo el forraje consumido en el pastoreo y el suplemento consumido durante el ordeño, no presentaron diferencias estadísticas significativas ( $\mathrm{P}>0,05)$, y se encontró dentro de valores normales de consumo para vacas lecheras en este tipo de sistema productivo, donde reciben suplementos balanceados (Baudracco et al., 2010; Cerdas, 2013).

El CMS durante el pastoreo para C25 fue menor que para C15, siendo importante relacionarlo con la mayor inclusión de botón de oro, dados los mayores niveles de FDN y FDA presentes en este suplemento en comparación con los otros dos; la evaluación realizada en el banco forrajero empleado para este estudio mostró niveles bajos de taninos totales (entre $0,08 \%$ y $0,11 \%$ ), nivel que no tendría efecto negativo sobre CMS (Min et al., 2003).

No se conocen reportes en vacas suplementadas con alimentos concentrados que contengan harina de botón de oro; no obstante, hay estudios en los que se incluyó $T$. diversifolia en la dieta de los animales, tales como el de Ribeiro et al. (2015) quienes encontraron un consumo de materia seca entre 18,7 kg y 18,9 kg por día, en vacas holstein cebú que recibieron dietas mixtas totales (TMR) que incluyeron 6,5\% y 15,4\% de la MS total de la dieta de hojas frescas de T. diversifolia. Los valores encontrados en este estudio estuvieron entre los valores encontrados por Escobar y Carulla (2003), quienes demostraron que el CMS puede variar entre 12,71 kg y hasta 23,47 kg por vaca al día, esto en función de la oferta de forraje. Al utilizar la FDAi (fibra detergente ácido insoluble) como marcador se encontró un CMS de 18,3 kg (Correa et al., 2009).

Es importante considerar el efecto de la suplementación y la composición del suplemento alimenticio sobre el consumo durante el pastoreo; Valencia (2013) encontró un CMS total de 18,5 kg y un consumo de 12,6 kg de MS de forraje cuando las vacas pastorearon en praderas de kikuyo y recibieron suplemento, en ambos casos, los resultados fueron similares a los hallados en este estudio. Sin embargo, es importante reconocer que la dieta que contenía el mayor nivel de harina de botón de oro podría llegar a ser un limitante para el CMS, debido al mayor llenado ruminal; de igual manera, la dieta con $25 \%$ de harina de $T$. diversifolia fue la que contuvo la mayor cantidad de FDN, lo que podría cambiar el tiempo de retención del alimento dentro del rumen y, de esta manera, hacer que se presenten cambios en la disponibilidad de la proteína y de la energía de la dieta (Bayati et al., 2013). 
El CMS bajo condiciones de pastoreo, puede ser un limitante en los sistemas de producción lechera especializada en el trópico alto, sobre todo si se considera un pasto de alto contenido de humedad como base de la alimentación forrajera, como el pasto kikuyo que en este estudio presentó niveles de $86,62 \%$, por lo que, este forraje al ser más voluminoso hace que se presente un llenado más rápido del rumen. De ahí, la importancia de encontrar un buen suplemento alimenticio que ayude a compensar las necesidades nutricionales del ganado lechero, pero que no afecte ni su desempeño productivo ni su salud.

\section{Cantidad y calidad de leche}

La cantidad de leche se evaluó a partir de la producción total durante todo el ensayo, la producción media estuvo influenciada por la producción de leche en la fase pre-experimental, que fue medida como covariable $(\mathrm{P}<0,05)$ (Cuadro 4). La producción de leche corregida al 4\% de grasa no presentó diferencias significativas (Cuadro 4).

Cuadro 4. Producción de leche, cantidad de grasa y proteína en la leche de vacas que consumieron suplementos con $0 \%$, $15 \%$ y $25 \%$ de harina de botón de oro (C0, C15 y C25), en la hacienda La Montaña, municipio de San Pedro de los Milagros, Antioquia, Colombia. 2015.

Table 4. Milk production, quantity of milk fat and protein of cows that consumed supplements with $0 \%, 15 \%$ and $25 \%$ of mexican sunflower flour (C0, C15 and C25), in the farm La Montaña, Municipality of San Pedro de los Milagros, Antioquia, Colombia. 2015.

\begin{tabular}{llccc}
\hline & & C0 & C15 & C25 \\
\hline Producción de leche $(\mathrm{kg})$ & Pre-experimental & $26,00 \mathrm{a}$ & $25,08 \mathrm{a}$ & $25,00 \mathrm{a}$ \\
& Fase experimental & $24,45 * \mathrm{a}$ & $24,28 * \mathrm{a}$ & $24,67 * \mathrm{a}$ \\
& Leche corregida (4\% grasa) & $21,99 \mathrm{a}$ & $22,45 \mathrm{a}$ & $21,89 \mathrm{a}$ \\
Composición de la leche & Producción de grasa $(\mathrm{kg})$ & $0,84 \mathrm{a}$ & $0,86 \mathrm{a}$ & $0,82 \mathrm{a}$ \\
& Producción de proteína $(\mathrm{kg})$ & $0,67 \mathrm{a}$ & $0,65 \mathrm{a}$ & $0,66 \mathrm{a}$ \\
& Grasa (\%) & $3,68 \mathrm{a}$ & $3,64 \mathrm{a}$ & $3,44 \mathrm{a}$ \\
& Proteína (\%) & $2,92 \mathrm{a}$ & $2,73 \mathrm{a}$ & $2,78 \mathrm{a}$ \\
\hline
\end{tabular}

Letras diferentes en la misma fila son significativamente diferentes $(\mathrm{p}<0,05) /$ Different letter in the same row indicated significant differences $(\mathrm{p}<0.05)$.

* Diferencia significativa con su covariable de la fase pre-experimental / Significant difference with covariable of the preexperimental period.

Al realizar un análisis de covariables, se encontró diferencia significativa para la calidad de leche obtenida en la fase experimental, cantidad de grasa y de proteína producida $(\mathrm{p}<0,05)$, lo que determinó que existió un cambio para estas variables como efecto del suplemento alimenticio suministrado.

La calidad de la leche obtenida durante la fase experimental se estimó según la composición porcentual y el volumen producido diariamente por cada vaca. Los valores medios de grasa y proteína no presentaron diferencias estadísticas significativas $(\mathrm{P}>0,05)$ (Cuadro 4).

La respuesta esperada a la suplementación alimenticia es el incremento en la producción de leche, sin embargo, esta puede ser limitada en cuanto al contenido de proteína y de grasa como consecuencias de la alta suplementación con alimentos ricos en almidones (Bargo et al., 2003) o por la falta de sincronía entre carbohidratos y nitrógeno que ingresan al rumen (Arroyave y Gallego, 2008). El suministro de los suplementos generalmente se realiza en dos 
raciones por día, tal como se hizo en este estudio, por lo que, se debe controlar su ofrecimiento a los animales, ya que el exceso puede llegar a generar otras dificultades como un descenso en el $\mathrm{pH}$ ruminal, que a su vez podría afectar la actividad microbiana, particularmente de las bacterias proteolíticas y celulolíticas, afectar su actividad enzimática y conllevar a situaciones como un menor crecimiento microbiano y menor producción de ácidos grasos volátiles.

La producción total de leche por día, fue similar entre los tres tratamientos y estuvo entre rangos normales para vacas de alta producción que llevan más de 70 días de lactancia. Se han encontrado entre 21,4 kg y 23,0 kg de leche ajustada, para vacas holstein que consumieron $18,9 \mathrm{~kg}$ de kikuyo y $5,9 \mathrm{~kg}$ de un suplemento de tipo tradicional

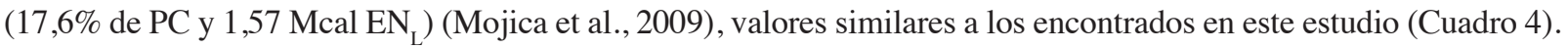

En lo referente a la calidad de la leche obtenida, se hallaron valores similares a los que se presentan de manera cotidiana en lecherías de trópico alto en Colombia (Mojica et al., 2009; Valencia, 2013).

Aunque no se presentaron diferencias estadísticamente significativas, la leche obtenida en este estudio se clasificó como una leche buena por el nivel de grasa, y de regular calidad por su valor proteico y de sólidos totales, de acuerdo con la clasificación de calidad de leches para Colombia (Calderón et al., 2006), donde, si los valores de grasa están entre 3,3\% y 3,5\% puede clasificarse como buena y si los valores están entre 2,6\% y 2,8\% para proteína y entre $11,3 \%$ y $11,8 \%$ para sólidos totales respectivamente, se le clasifica como regular.

Con respecto a la grasa láctea, en porcentaje o total producida, no se presentaron diferencias significativas. Los valores de grasa encontrados en este estudio fueron superiores al 3,31\% encontrado en dietas en las que se empleó un suplemento tradicional (Valencia, 2013).

La proteína láctea mostró valores aceptables en el mercado (Calderón et al., 2006). El nivel de proteína pudo estar influenciado por los carbohidratos solubles que se emplearon en la formulación de los suplementos, que disminuyeron en la medida que se aumentó $T$. diversifolia en el suplemento, material que reemplazó en parte al maíz extruido (Cuadro 1); asimismo, los compuestos fibrosos contenidos en el botón de oro, pudieron incidir en la eficiencia con que se emplea el amoníaco ruminal para la síntesis de proteína microbiana, ya que cuando el tiempo de retención del alimento es mayor en el rumen la proteína puede aumentar su degradabilidad, con la consecuente pérdida de nitrógeno amoniacal (Lazzarini et al., 2009). Se han reportado efectos sobre la concentración de la proteína en función del consumo de materia seca (Bargo et al., 2003; Escobar y Carulla, 2003).

Las concentraciones de nitrógeno ureico en leche fueron mayores a $18 \mathrm{mg} / \mathrm{dl}$ para los tres tratamientos; si el MUN es superior a $18 \mathrm{mg} / \mathrm{dl}$ y la proteína de la leche es menor que 3,0\% (Acosta et al., 2005), como se presentó en este estudio (Cuadro 5), la dieta podría estar excediendo su proteína soluble o degradable en relación con la disponibilidad de carbohidratos fermentables. Los niveles de MUN se asocian con el balance y sincronización entre la energía y la proteína que ingresan al rumen (Arroyave y Gallego, 2008).

$\mathrm{Si}$ bien las dietas fueron isoenergéticas e isoproteicas, es posible que no se haya dado una adecuada sincronía entre la proteína degradable en rumen y la disponibilidad energética, en términos del tiempo requerido para su aprovechamiento por parte de las bacterias ruminales; los carbohidratos totales fueron altos en la dieta (kikuyo $63,59 \%)$ y similares en los tres suplementos (C0:74,68\%, C15:76,41\% y C25:74;18\%), sin embargo, para C25 la cantidad de carbohidratos no fibrosos fue inferior (C0:58,81\%, C15:58,50\% y C25:48,50\%). Ante una deficiencia de carbohidratos solubles las bacterias no pueden convertir el amoníaco en proteína microbiana, por lo que, puede aumentar su paso a sangre (Lazzarini et al., 2009).

Frente a un mayor paso de proteína hacia el intestino delgado, es posible que se presente desaminación de los aminoácidos absorbidos a nivel hepático, en especial por un menor balance energético en aquellas vacas con mayores niveles de producción; en ambos casos este amoníaco incidiría en el aumento de MUN (González y Koenekamp, 2006).

Un estudio mostró que los niveles de MUN pueden estar más influenciados por la cantidad de proteína degradable en rumen que con la proteína total de la dieta, por lo que, es necesario estudiar el fraccionamiento de la 
Cuadro 5. Calidad de la leche de vacas que consumieron suplementos que contenían $0 \%, 15 \%$ y $25 \%$ de harina de botón de oro (C0, C15 y C25), en la hacienda La Montaña, municipio de San Pedro de los Milagros, Antioquia, Colombia. 2015.

Table 5. Milk quality of cows that consumed supplements with $0 \%, 15 \%$ and $25 \%$ of mexican sunflower flour (C0, C15 and C25), in the farm La Montaña, Municipality of San Pedro de los Milagros, Antioquia, Colombia. 2015.

\begin{tabular}{lccc}
\hline Parámetro & C0 & C15 & C25 \\
\hline Lactosa & $4,46 \mathrm{a}$ & $4,64 \mathrm{~b}$ & $4,63 \mathrm{~b}$ \\
Sólidos totales & $11,86 \mathrm{a}$ & $11,79 \mathrm{a}$ & $11,61 \mathrm{a}$ \\
Recuento de células somáticas (RCS) & $177,08 \mathrm{a}$ & $80,08 \mathrm{a}, \mathrm{b}$ & $44,08 \mathrm{~b}$ \\
Nitrógeno ureico en leche (MUN) & $18,56 \mathrm{a}$ & $22,88 \mathrm{~b}$ & $24,11 \mathrm{~b}$ \\
\hline
\end{tabular}

Letras diferentes en la misma fila son significativamente diferentes $(p<0,05)$ / Different letter in the same row indicate significant differences $(\mathrm{p}<0.05)$.

proteína del botón de oro y, según su capacidad de disolución, acompañar los suplementos con materias primas que contengan carbohidratos que presenten diferente solubilidad, lo que permitiría mejorar la sincronía entre nitrógeno y carbohidratos en el rumen (Baker et al., 1995); una opción podría ser la utilización de bloques multinutricionales de melaza sin urea, ofrecidos directamente en el potrero.

La relación PC:EN $\mathrm{L}_{\mathrm{L}}$ en la dieta fue de C0:110,95, C15:116,19 y C25:115,15 g/Mcal EN $\mathrm{L}_{\mathrm{L}}$; cuando esta relación es superior a 105, el MUN podría ser superior a $20 \mathrm{mg} / \mathrm{dl}$ (Hess et al., 1999), tal como se dio en este caso.

Las fuentes de proteína empleadas en este estudio tienen diferentes sitios de degradación, por la baja cantidad de taninos en el botón de oro empleado, su proteína debe considerarse degradable en rumen, lo que podría ser una de las causas del incremento del nitrógeno ureico en leche (Wang et al., 2009; Lee et al., 2011).

Las vacas con más producción de leche también presentan mayor MUN, esto se asocia al mayor gasto de aminoácidos para la obtención de energía (Khon, 2007). De acuerdo con Gustafsson y Palmquist (1993), existen fluctuaciones en los niveles de urea a lo largo del día, debido al pastoreo o diferentes momentos y tipos de suplementación, por lo que, el fraccionamiento del suplemento, no solo en dos veces como se hizo en este trabajo, sino en varias tomas a lo largo del día, o el uso de sistemas silvopastoriles con ramoneo de botón de oro, permitirían mejorar la sincronía entre carbohidratos y nitrógeno en el rumen.

La lactosa encontrada presentó diferencias significativas entre $\mathrm{C} 0$ y $\mathrm{C} 15$ y C25, pero no entre estos dos últimos (Cuadro 5); de acuerdo con estos niveles, la leche encontrada fue regular para C15 y C25 y mala para C0 (Calderón et al., 2006), la cantidad de lactosa juega un papel de gran importancia en la determinación del volumen de leche que llegue a producirse (Campabadal, 1999). La lactosa producida depende de la glucosa que sea posible retener en la glándula mamaria y del estado de salud de esta; cuanto mayor es el número de células somáticas en la leche, menor es el contenido de lactosa (Ramos et al., 2015).

En el presente trabajo se obtuvieron niveles en el recuento de células somáticas (RCS) considerados como buenos para C15 y C25 y malos para C0, según las tablas de calificación de leches para Colombia (Valencia, 2013). Los menores niveles en RCS se dieron al incrementar la inclusión de harina en el suplemento; esta situación puede estar asociada con el contenido de metabolitos secundarios como los sesquiterpenos de lactona, los que presentan un importante efecto antiinflamatorio (Chagas et al., 2011). 


\section{Relación beneficio:costo}

La relación entre la leche producida y la cantidad de suplemento consumido en cada tratamiento fue mayor para C25 con diferencias estadísticamente significativas (Cuadro 6). La relación beneficio:costo presentó diferencias altamente significativas $(\mathrm{p}<0,001)$ entre los tratamientos y la mejor relación entre el costo del alimento y el precio de la leche producida fue para C25 (Cuadro 6).

Cuadro 6. Relación entre la leche producida, suplemento y precio del suplemento con diferentes niveles de harina de botón de oro $(\mathrm{C} 0, \mathrm{C} 15$ y C25) suministrado a vacas de alta producción, en la hacienda La Montaña, municipio de San Pedro de los Milagros, Antioquia, Colombia. 2015.

Table 6. Relationship between milk yield, supplement and supplement price with different levels of mexican sunflower flour (C0, C15 y C25) supplied to high production cows, in the farm La Montaña, Municipality of San Pedro de los Milagros, Antioquia, Colombia. 2015.

\begin{tabular}{lccc}
\hline Parámetro & C0 & C15 & C25 \\
\hline Relación leche:suplemento & $3,12 \mathrm{a}$ & $3,85 \mathrm{~b}$ & $4,38 \mathrm{c}$ \\
Precio suplemento $\left(\$_{\mathrm{COP}} / \mathrm{kg}\right)$ & 816,95 & 796,68 & 725,35 \\
Relación beneficio:costo & $2,96^{*}$ & $3,71 * *$ & $4,60 * * *$ \\
\hline
\end{tabular}

Letras diferentes en la misma fila son significativamente diferentes $(\mathrm{p}<0,05)$ / Different letter in the same row indicate significant differences $(\mathrm{p}<0.05)$.

* Diferencias altamente significativas $(\mathrm{p}<0,01) /$ Highly significant differences $(\mathrm{p}<0.01)$.

La relación entre la leche producida y el suplemento consumido encontrado, mostró que, cuando se utilizaron suplementos que incluyeron harina de $T$. diversifolia, se mejoró la eficiencia productiva de los animales; si a esto se le suma que el costo por kilogramo de suplemento fue menor en la medida que se aumentó la harina de botón de oro, se generan aún mejores expectativas para la implementación de esta especie forrajera como materia prima en la formulación de alimentos balanceados.

La relación beneficio:costo estimada entre el precio de venta por litro de leche, sin incluir ningún tipo de bonificación, y el costo por kilogramo de suplemento alimenticio suministrado, dejó en gran ventaja a C25 con respecto a $\mathrm{C} 15$ y más aún frente a $\mathrm{C} 0$. Sin embargo, deben estimarse las posibles bonificaciones a las que se puedan acceder y, la calidad higiénica y RCS, que en este caso también fueron mejores para C25 y C15 con respecto a C0; la bonificación por RCS puede llegar a significar hasta un $20 \%$ más en el precio de la leche.

\section{Conclusiones}

La inclusión de niveles de hasta el $25 \%$ de harina de Tithonia diversifolia como ingrediente en la elaboración del suplemento alimenticio, suministrado a vacas lecheras manejadas en praderas de kikuyo en el trópico alto, no afectó el volumen de leche, la cantidad de proteína y grasa producidas, y mejoró la calidad microbiológica de la leche, en lo referente al recuento de células somáticas. La inclusión creciente da la harina de botón de oro hasta el $25 \%$, permitió mejorar la relación entre el suplemento consumido y la leche producida, con un importante efecto sobre la relación beneficio:costo. Se recomienda evaluar la harina de botón de oro bajo otras condiciones de manejo, empleando diferentes materias primas en la elaboración del suplemento alimenticio. 


\section{Agradecimientos}

Los autores agradecen al Proyecto de Sostenibilidad 2011-2012 (CODI, Universidad de Antioquia) y al proyecto CODI mediana cuantía 2011 Acta CODI 614 del 14/02/12, por el apoyo económico para la ejecución de este trabajo.

\section{Literatura citada}

Acosta, Y., I. Delucchi, M. Olivera, y C. Diesta. 2005. Urea en leche: factores que la afectan. Instituto Nacional de Investigación Agropecuaria (INIA), Sitio Argentino de Producción Animal.http://www.produccion-animal.com.ar/produccion_bovina_de_ leche/leche_subproductos/56-urea_en_leche.pdf (consultado 12 nov. 2015).

AOAC (Association of Official Analytical Chemists). 2005. Official methods of analysis of AOAC International. 18 ${ }^{\text {th }}$ ed. AOAC Int., MD, USA.

Arroyave, E., y L. Gallego. 2008. Análisis de la sincronía entre energía y proteína en vacas lactantes y su relación con la eficiencia en la síntesis de proteína microbial. Tesis Esp., Universidad de Antioquia, Medellín, COL.

Baker, 1., J. Ferguson, and W. Chalupa. 1995. Responses in urea and true protein of milk to different protein feeding schemes for dairy cows. J. Dairy Sci. 78:2424.

Bargo, F., L.D. Muller, E.S. Kolver, and J.E. Delahoy. 2003. Invited review: production and digestion of supplemented dairy cows on pasture. J. Dairy Sci. 86:1-42.

Bayati, J., Z. Moradi, and N. Moradi. 2013. Synchronization of energy and protein on supply synthesis microbial protein. Int. J. Adv. Biol. Biomed. Res. 1:594-600.

Buxadé, C. 1995. Zootecnia bases de producción animal: reproducción y alimentación. Tomo II. Ediciones Mundiprensa, Madrid, ESP.

Baudracco, J., N. Lopez-Villalobos, C. Holmes, and K. Macdonald. 2010. Prediction of herbage dry matter intake for dairy cows grazing ryegrass-based pastures. Proc. New Zealand Soc. Anim. Prod. 70:80-85.

Boschini, C., y L. Pineda. 2016. Ensilaje de kikuyo (Pennisetum clandestinum o Kikuyuocloa clandestina) fermentado con tres aditivos. Agron. Mesoam. 27:49-60.

Calderón, A., F. García, y G. Martínez. 2006. Indicadores de calidad de leches crudas en diferentes regiones de Colombia. Rev. MVZ Córdoba 11:725-737.

Campabadal, C. 1999. Factores que afectan el contenido de sólidos de la leche. Nutr. Anim. Trop. 5:67-92.

Cerdas, R. 2013. Formulación de raciones para carne y leche. Desarrollo de un módulo práctico para técnicos y estudiantes de ganadería de Guanacaste, Costa Rica. Intersedes 29:128-153.

Chagas, D., R. Barbosa, V. da Silva, L. Gobbo, T. Gasparotoc, A. Campanellic, L. Faccioli, and F. Batista. 2011. Chlorogenic acids from Tithonia diversifolia demonstrate better anti-inflammatory effect than indomethacin and its sesquiterpene lactones. J. Ethnopharmacol. 136:355-362.

Correa, H., M. Pabón, y J. Carulla. 2009. Estimación del consumo de materia seca en vacas Holstein bajo pastoreo en el trópico alto de Antioquia. Liv. Res. Rural Dev. 21(4):59. http://www.lrrd.org/lrrd21/4/corr21059.htm (consultado 9 nov. 2013).

Correa, H., Y. Rodríguez, M. Pabón, y J. Carulla. 2012. Efecto de la oferta de pasto kikuyo (Pennisetum clandestinum) sobre la producción, la calidad de la leche y el balance de nitrógeno en vacas Holstein. Liv. Res. Rural Dev. 24(11):204. http://www. lrrd.org/ lrrd24/11/corr24204.htm (consultado 19 nov. 2016). 
Escobar, A., y J. Carulla. 2003. Efecto de la oferta de forraje sobre los parámetros productivos y composicionales de la leche en la Sabana de Bogotá. Rev. Colom. Cienc. Pecu. 16:74.

Gallego, L., L. Mahecha, y J. Angulo. 2015. Crecimiento y desarrollo de Tithonia diversifolia Hemsl. A Gray en condiciones de trópico alto. En: P. Pietri, editor, $3^{\circ}$ Congreso Nacional de Sistemas Silvopastoriles, VIII Congreso Internacional de Sistemas Agroforestales, Misiones, Argentina. Santa Cruz Ediciones, INTA, ARG. p. 53-57.

González, F., y I. Koenekamp. 2006. Adaptaciones metabólicas hepáticas en el período periparto en vacas de alta producción de leche. Pontificia Universidad Católica de Chile, CHI.

Gualberto, R., O. Souza, N. Costa, C. Braccialli, e L. Gaion. 2010. Influência do espaçamento e do estádio de desenvolvimento da planta na produção de biomassa e valor nutricional de Tithonia diversifolia (hemsl.) Gray. Nucleus 7(2):135-149.

Gustafsson, A., and D. Palmquist. 1993. Diurnal variation of rumen ammonia, serum urea and milk urea in dairy cows at high and low yields. J. Dairy Sci. 76:475.

Hess, H.D., H. Flórez, C.E. Lascano, L.A. Baquero, A. Becerra, y J. Ramos. 1999. Fuentes de variación en la composición de la leche y niveles de urea en sangre y leche de vacas en sistemas de doble propósito en el trópico bajo de Colombia. Pasturas Trop. 21:33-42.

Khon, R. 2007. Use of milk or blood urea nitrogen to identify feed management inefficiencies and estimate nitrogen excretion by dairy cattle and other animals. Paper presented at: $18^{\text {th }}$ Annual Florida Ruminant Nutrition Symposium. Paper 8. University of Florida, 30-31 Jan. Gainesville, FL, USA.

Lachmann, M., O. Araujo, y J. Vergara. 2003. Evaluación de la lignina detergente ácido como marcador para la determinación de la digestibilidad en ovinos. Rev. Científica FCV-LUZ 13:484-489.

Lazzarini, I., E. Detmann, C. Batista, M. Fonseca, S. de Campos, M. Augusto, and F. Albani. 2009. Intake and digestibility in cattle fed low-quality tropical forage and supplemented with nitrogenous compounds. Rev. Bras. Zootec. 38:2021-2030.

Lee, S., S.M. Lee, Y. Cho, D. Kam, S.C. Lee, C. Kim, and S. Seo. 2011. Glycerol as a feed supplement for ruminants: In vitro fermentation characteristics and methane production. Anim. Feed Sci. Technol. 166-167:269-274.

Lugo, S., F. Molina, I. González, J. González, y E. Sánchez. 2012. Efecto de la altura y frecuencia de corte sobre la producción de materia seca y proteína cruda de Tithonia diversifolia (Hemsl) A. Gray. Zootec. Trop. 30:317-325.

Macías, M., y O. Martínez. 1997. Composición en aminoácidos de diferentes fuentes tropicales no convencionales para la alimentación animal. Rev. Computarizada Prod. Porcina 4(3):1-60.

Mahecha, L., J. Escobar, J. Suárez, y L. Restrepo. 2007. Tithonia diversifolia (hemsl.) Gray (botón de oro) como suplemento forrajero de vacas F1 (Holstein por Cebú). Liv. Res. Rural Dev. 19(2):16. http:// www.lrrd.org/lrrd19/2/mahe19016.htm (consultado 9 nov. 2013).

Mahecha, L., L. Gallego, y F. Peláez. 2002. Situación actual de la ganadería de carne en Colombia y alternativas para impulsar su competitividad y sostenibilidad. Rev. Colom. Cienc. Pecu. 15:213-225.

Maina, I., S. Abdulrazak, C. Muleke, and T. Fujihara. 2012. Potential nutritive value of various parts of wild sunflower (Tithonia diversifolia) as source of feed for ruminants in Kenya. J. Food Agric. Env. 10:632-635.

Medina, M., D. García, E. González, L. Cova, y P. Morantinos. 2009. Variables morfo-estructurales y de calidad de la biomasa de Tithonia diversifolia en la etapa inicial de crecimiento. Zootec. Trop. 27:121-134.

Min, B., T. Barry, G. Attwood, and W. McNabb. 2003. The effect of condensed tannins on the nutrition and health of ruminants fed fresh temperate forages. Anim. Feed Sci. Technol. 106:3-19. 
Mojica, J., E. Castro, J. León, E. Cárdenas, M. Pabón, y J. Carulla. 2009. Efecto de la oferta de pasto kikuyo y ensilaje de avena sobre la producción y calidad composicional de la leche bovina. Corpoica Cienc. Tec. Agropecu. 10:81-90.

Morales, A., J. León, E. Cárdenas, G. Afanador, y J. Carulla. 2013. Calidad de la leche, digestibilidad in vitro de la materia seca y producción de vacas alimentadas con gramíneas solas o asociadas con Lotus uliginosus. Rev. Med. Vet. Zootec. 60:32-48.

NRC (National Research Council). 2001. Nutrient requirements of dairy cattle. $7^{\text {th }}$ ed. NRC, WA, USA.

Nieves, D., O. Terán, L. Cruz, M. Mena, F. Gutiérrez, y J. Ly. 2011. Digestibilidad de nutrientes en follaje de árnica (Tithonia diversifolia) en conejos de engorde. Trop. Subtrop. Agroecosyst. 14:309-314.

Ramos, R., V. Bufon, K. Molin, E. Walter, M. Rezende, R. Fagnani, and A. Ludovico. 2015. Relationship between somatic cell counts and milk production and composition in jersey cows. Rev. Salud Anim. 37:137-142.

Ribeiro, R., S. Terry, J. Sacramento, S. Rocha, C. Pereira, E. Fernandes, H. Cuquetto, M. Sundfeld, L. Ribeiro, T. Ribeiro, R. Martins, and A. Vieira. 2015. The effects of Tithonia diversifolia on dairy cow performance. PLoS ONE 11:1-18.

Rivera, J., C. Cuartas, J. Naranjo, O. Tafur, E. Hurtado, F. Arenas, J. Chará, y E. Murgueitio. 2015. Efecto de la oferta y el consumo de Tithonia diversifolia en un sistema silvopastoril intensivo (SSPi), en la calidad y productividad de leche bovina en el piedemonte Amazónico colombiano. Liv. Res. Rural Dev. 27(10):189. http://www.lrrd.org/lrrd27/10/ rive27189.html (consultado 19 nov. 2016).

Valencia, D. 2013. Efecto de la suplementación de dietas para vacas lecheras con glicerina cruda, sobre algunos parámetros de la fermentación ruminal, producción y calidad composicional de la leche. Tesis Magister en Ciencias Agrarias, Universidad Nacional de Colombia, COL.

Wang, C., Q. Liu a, W. Huo, W. Yang, K. Dong, Y. Huang, and G. Guo. 2009. Effects of glycerol on rumen fermentation, urinary excretion of purine derivatives and feed digestibility in steers. Liv. Sci. 121:15-20. 